\title{
Thermal stabilities and optimal operating parameters for the Oak Ridge Spallation Neutron Source superconducting linear accelerator
}

\author{
Sang-ho Kim and Isidoro E. Campisi \\ Oak Ridge National Laboratory, Oak Ridge, Tennessee 37831, USA
}

(Received 12 September 2006; published 28 March 2007)

\begin{abstract}
The baseline Spallation Neutron Source (SNS) accelerator will provide a 1-GeV, 1.4-MW proton beam to a mercury target for the production of neutrons. The main acceleration for the H-beam is provided by 81 superconducting cavities installed in 23 cryomodules operating at $805 \mathrm{MHz}$. The design of the superconducting linac includes a $2.1-\mathrm{K}, 2.5-\mathrm{kW}$ cryogenic plant to maintain the cavities below the helium lambda point for efficient operation at high accelerating gradients. In this paper operating conditions are analyzed rather than the design ones, which still guarantees a high gradient operation without any temperature constraint. From the analysis it appears that the SNS superconducting linac can be operated at temperatures higher than $2.1 \mathrm{~K}$, a fact resulting from both the pulsed nature of the superconducting cavities, the specific configuration of the existing cryogenic plant, and the operating frequency. General conditions are also given regarding the operation of pulsed superconducting cavities resonating at different frequencies.
\end{abstract}

DOI: 10.1103/PhysRevSTAB.10.032001

PACS numbers: 29.17.+w, 74.25.Op, 74.25.Bt, 74.25.Fy

\section{INTRODUCTION}

Eighty-one superconducting elliptical cavities built out of 4-mm thick niobium sheet provide acceleration to the $\mathrm{H}$ ion beam from $187 \mathrm{MeV}$ to about $1 \mathrm{GeV}$ in the Spallation Neutron Source (SNS) linac [1]. The cavities belong to two separate groups: 33 medium beta cavities (optimally matched to particles traveling at $\beta=0.61$ ) and 48 high beta $(\beta=0.81)$ cavities in 11 and 12 cryomodules, respectively. The cryomodules and all the components within them were designed, later processed, and assembled at Thomas Jefferson National Laboratory. Together with the cavities and cryomodules, the cryogenic plant and helium distribution system were also designed at JLab and installed at SNS [2,3]. The design operating temperature of the system is $2.1 \mathrm{~K}$, just below the helium superfluid transition at $2.17 \mathrm{~K}$, the lambda point. The choice of temperature guaranteed that the BCS surface resistance at $805 \mathrm{MHz}$ would be equivalent to standard accepted values of the residual resistance; that the radio-frequency (RF) surface dissipations would be comparable to the load into the cryogenic system induced by field-emission currents; and that heat generated by RF and field emissions would be efficiently removed by the superfluid helium.

Because of unforeseen circumstances, the availability of the cryogenic plant's subatmospheric system was delayed past the installation of most of the cryomodules. The necessity arose for testing cavities and cryomodules in the SNS tunnel as soon as it was possible, in order to determine their acceptable functional characteristics. Without the subatmospheric helium system operating, it was decided to initiate testing of the systems at $4.2 \mathrm{~K}$, with tests to be later completed and validated once the complete cryogenic system would be capable of 2.1-K operation.
The superconducting cavities in the SNS linac have the characteristic of being powered in a pulsed mode (1.3msec pulses at 60 pulses per second), which is unique among superconducting accelerators now in routine operation. At first, the pulsed operation simply decreases the average dynamic heat load on the cryogenic system, but it was also known that, for sufficiently short pulses, the maximum operating gradients of a superconducting cavity are not necessarily achieved in liquid helium, much less in superfluid helium [4].

As the testing of the superconducting cavities progressed, it became apparent that the gradients that could be achieved at $4.2 \mathrm{~K}$ not only met the original design values required for SNS operation, but also exceeded them in a significant number of cavities [5]. Once the subatmospheric part of the cryogenic plant became operational, additional tests of the maximum achievable gradients in the superconducting cavities clearly indicated that the limiting factors in the cavities were marginally, if at all, related to the operating temperature [6].

At the initially limited beam power required by the SNS commissioning plan, the RF repetition rate was limited to 10 pulses per second and beam operations became possible even with the cavities being operated at $4.2 \mathrm{~K}$. This result is unprecedented in the history of commissioning and operation of superconducting RF accelerators.

The experimental results stimulated us to evaluate the details of the pulsed operation of the SNS Superconducting Linac (SCL), in order to establish operating parameters and criteria which would help to make the best use of the available equipment during normal operation, as well as guaranteeing a minimal performance of the machine under off- normal circumstances; for instance, during a failure of the subatmospheric cryogenic system. 
TABLE I. Major parameters of the SNS cavities at the designed accelerating gradient.

\begin{tabular}{lcc}
\hline \hline \multicolumn{1}{c}{ Parameter } & Medium beta cavity & High beta cavity \\
\hline Geometric beta & 0.61 & 0.81 \\
$E_{o} T(\mathrm{MV} / \mathrm{m})$ & $10.1($ at $\beta=0.61)$ & $15.9($ at $\beta=0.81)$ \\
$E_{\text {peak }}(\mathrm{MV} / \mathrm{m})$ & 27.5 & 35.0 \\
$H_{\text {peak }}(\mathrm{mT})$ & 58 & 75 \\
$Q^{*} R_{s}(\Omega)$ & 176 & 228 \\
$r / Q(\Omega)$ at the geometric beta & 279 & 483 \\
Equivalent cavity length $(\mathrm{cm})$ & 68.2 & 90.6 \\
\hline \hline
\end{tabular}

In this paper we review the operating conditions of the SNS SCL, we evaluate the interplay of the cavity parameters, and we give details on the possible range of operating parameters which can be achieved at SNS in pulsed conditions, with and without consideration of efficiency. In fact, given the design of the cryogenic plant, the highest overall efficiency is not necessarily achieved when the nominally optimal thermodynamic conditions are reached. Since the cryogenic plant has to run at a fixed load no matter what the actual static and dynamic loads from the cryomodules, a more efficient use of the plant is in fact at temperatures different from the designed ones.

Besides giving optimal conditions for SNS, we analyze and evaluate possible optimal parameters of pulsed superconducting cavities operated at other frequencies under similar parameters to those of SNS.

\section{SNS CAVITY}

The SNS cavities were designed mainly to keep the peak RF surface fields below certain values, along with other concerns such as Lorentz force detuning and cell-to-cell
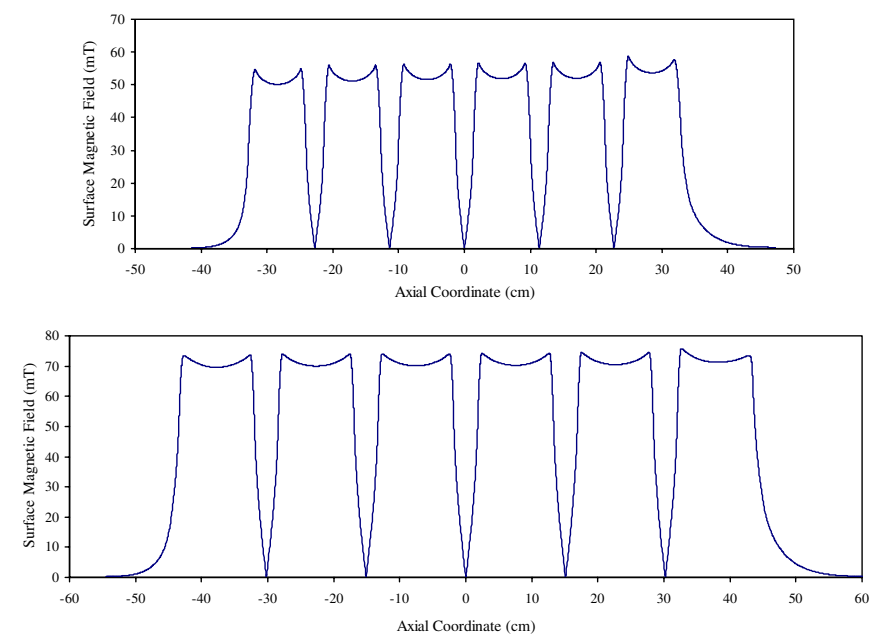

FIG. 1. (Color) Magnetic field distribution along the inner surface of the SNS medium beta cavity (top) and the SNS high beta cavity (bottom) at the nominal accelerating gradients 10 and 15.9 MV/m, respectively. The SNS cavities have six cells and " 0 " in the axial coordinate indicates the center of the cavity. coupling, etc. Table I summarizes the major parameters of the SNS superconducting radio-frequency (SRF) cavities. Under nominal operating conditions, the maximum surface magnetic field is about 58 and $75 \mathrm{mT}$ for the medium and high beta cavities, respectively, as shown in Fig. 1.

These values are much lower than the established limiting fields for niobium at the nominal operating temperature, which implies, in principle, an ample margin of operation and/or a possibility of operation even at temperatures higher than $2.1 \mathrm{~K}$. Presently, the highest maximum field observed in a SNS cavity is well over $130 \mathrm{mT}$ in a medium beta cavity, at both 4.2 and $2.1 \mathrm{~K}$.

\section{THERMAL STABILITY}

\section{A. Relations between physical parameters}

Relations of physical parameters for the thermal stability are shown in Fig. 2. Bulk thermal behaviors of SRF cavities can be expressed in terms of thermomagnetic bulk material

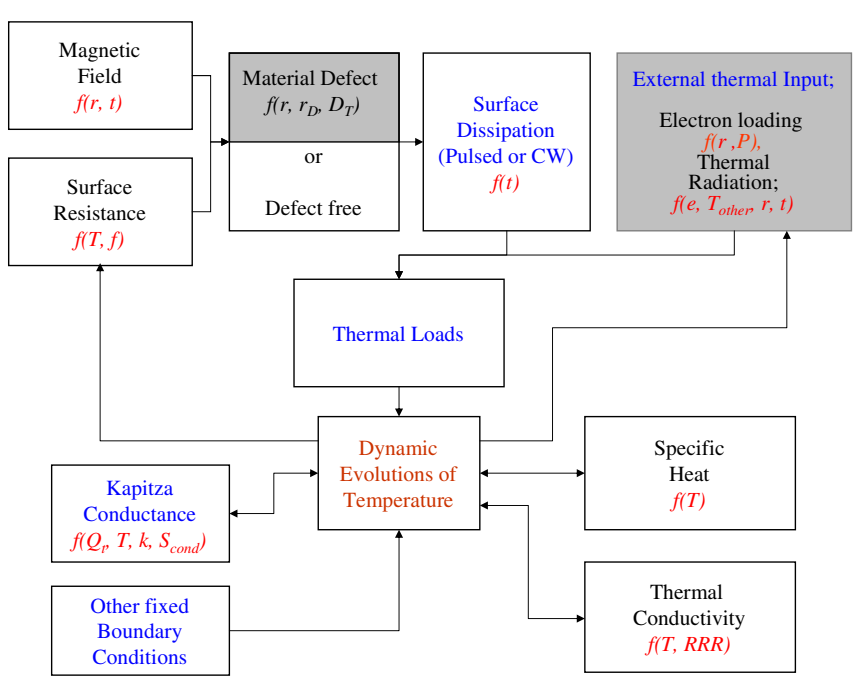

FIG. 2. (Color) Relations of physical parameters for the thermal stability in a SRF cavity [ $r$ : location; $t$ : time; $r_{D}$ : defect radius; $D_{T}$ : defect type; $P$ : external thermal load such as electron loading; $\varepsilon$ : emissivity; $T_{\text {other }}$ : temperatures of other structure; $R R R$ : residual resistivity ratio; $Q_{t}$ : thermal flux; $f$ : frequency; $k$ : thermal conductivity; $S_{\text {cond }}$ : surface condition; $T$ : temperature of niobium (gray-colored blocks are excluded in this analysis)]. 
properties such as residual resistivity ratio RRR, thermal conductivity $k$, specific heat $C_{p}$, heat transfer coefficient to helium $h_{k}$, and RF surface resistance $R_{s}$ at given conditions (for example, an operating frequency, a bath temperature of helium, surface magnetic fields, a thickness of niobium, etc.). In pulsed operation, time scales to build thermal stability and to cool down lengths in gaps are also a factor in connection with the repetition rate and pulse length.

In the following calculations, we neglect material defects since most cavities made of high RRR niobium are no longer affected by large defect dissipation, and we ignore electron loadings and the resulting heating. Whereas most cavities at SNS are affected and possibly limited by field emission-induced heating, it is difficult to apply uniform assumptions to all the cavities; while quenching patterns can be simulated by assuming a location, a field-emission induced heating power, etc. However, one can always find an operating gradient for which field emission does not constitute a limitation.

Thus the thermal loads in this study are RF surface dissipations that are given by $P=\frac{1}{2} R_{S} H^{2}$, where $P$ is the power dissipation per unit area in $\mathrm{W} / \mathrm{m}^{2}$ on the inner surface of a cavity, $R_{s}$ is the RF surface resistance in $\Omega$, and $H$ is the surface magnetic field strength on the inner surface of a cavity in A/m. The RF surface resistance is known to be originated from different mechanisms; BCS resistance, residual resistance, resistance due to trapped magnetic flux, resistance enhancement at around grain boundary, etc. Figure 3 shows the surface resistances from BCS and residual contributions at $805 \mathrm{MHz}$ and, for comparison, at $1300 \mathrm{MHz}$ where $10 \mathrm{n} \Omega$ of residual resistance is assumed. The assumed residual resistance is included mainly for calculations at lower temperatures $(T<2.5 \mathrm{~K})$ where the BCS and residual resistances are comparable. Other factors could be included in the assumed residual resistance or, if not, are neglected in the following study [7].

The BCS resistance is a highly nonlinear and strongly dependent material property. Figure 4 shows BCS surface dissipations per unit area for cavities operated at various surface magnetic fields at $805 \mathrm{MHz}$ (SNS) and $1300 \mathrm{MHz}$ (TESLA). The RF surface magnetic fields of 100, 130, and $170 \mathrm{mT}$, shown in Fig. 4, correspond to 21, 27.5, and $36 \mathrm{MV} / \mathrm{m}$ for the SNS high beta cavity and 23,30 , and $40 \mathrm{MV} / \mathrm{m}$ for the TESLA baseline cavity, respectively.

Thermal conductivities are known to have an approximate relation with RRR's, which is $k=\mathrm{RRR} / 4$ in $\mathrm{W} / \mathrm{m} / \mathrm{K}$ at $4.2 \mathrm{~K}$. Using this relationship could give misleading results when applied to other temperature regions in specific cases. Thermal conductivity $k$ versus temperature data for various RRR's in Fig. 5 are taken from [8-10], where the RRR values marked with RRR1 to RRR9 are 40, 270, 525, 90, 250, 400, 140,840, and 70, respectively. Presently niobium sheets having RRR about 200 or higher are most widely used for SRF cavities and industrial pro-

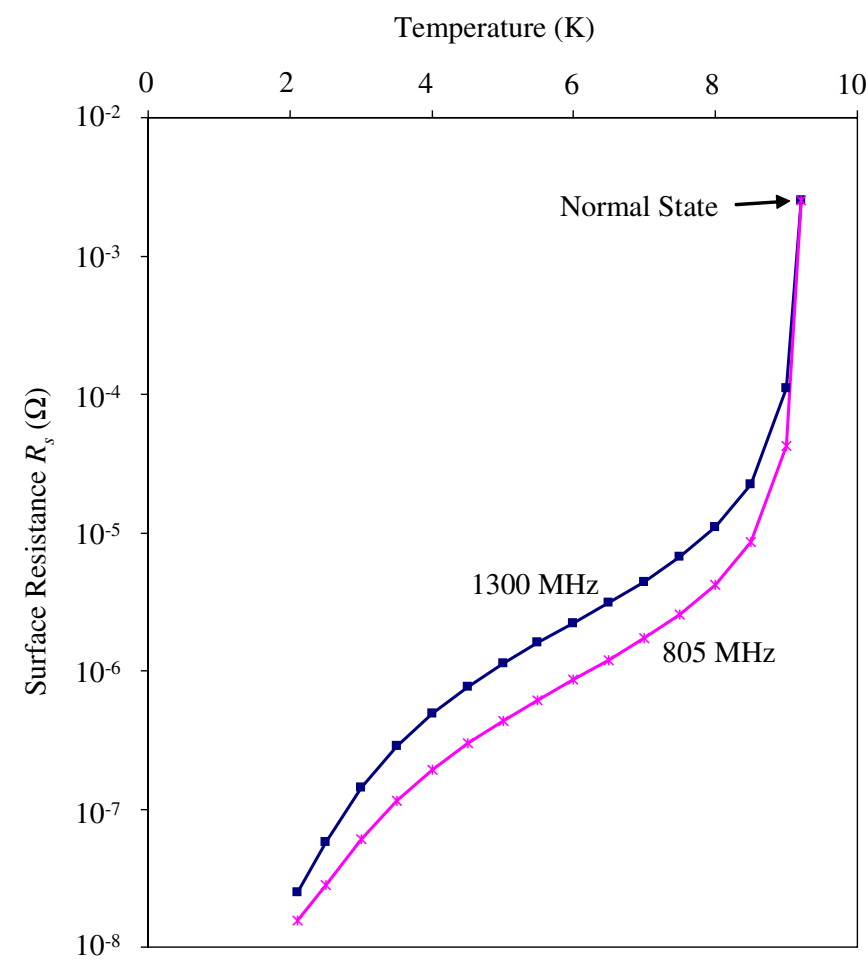

FIG. 3. (Color) RF surface resistances of niobium at 805 and $1300 \mathrm{MHz} .10 \mathrm{n} \Omega$ of residual resistances are assumed in this plot.

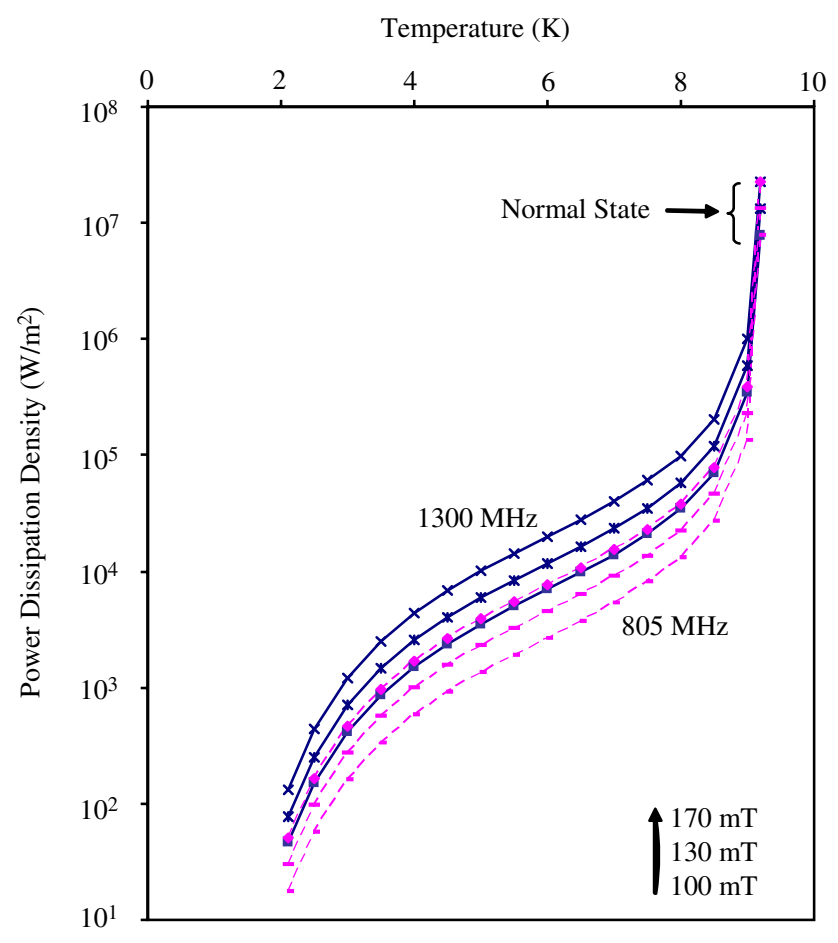

FIG. 4. (Color) Power dissipation densities on niobium surfaces calculated from the surface resistances and the surface magnetic fields. Dashed lines are surface power dissipations at 100, 130, and $170 \mathrm{mT}$ operated at $805 \mathrm{MHz}$ from the bottom. Solid lines are surface power dissipations at 100,130, and $170 \mathrm{mT}$ operated at $1300 \mathrm{MHz}$ from the bottom. 


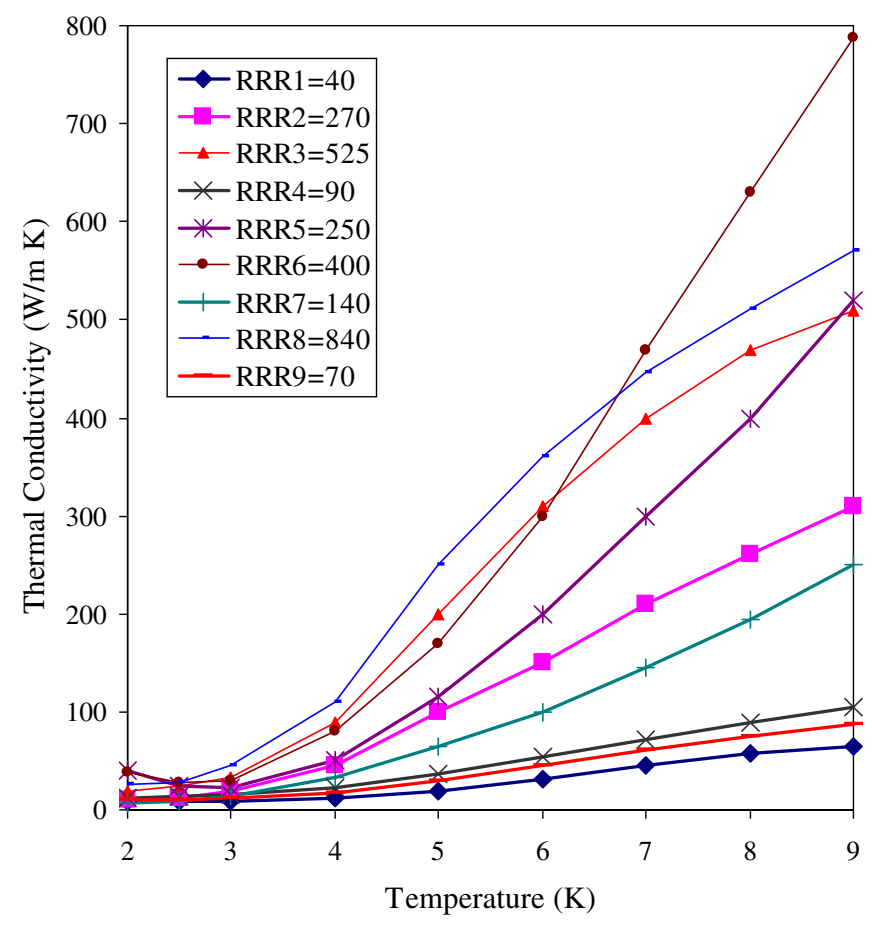

FIG. 5. (Color) Thermal conductivities of niobium for various RRR's taken from [8-10], where the residual resistivity ratio (RRR) values marked with RRR1 to RRR8 are 40, 270, 525, 90, $250,400,140$, and 840 , respectively. The end groups of the SNS cavities are made of reactor grade niobium and are heat treated to increase RRR up to 70 that is marked with RRR9 in this plot.

ductions are quite mature. RRR5 is taken for the following analysis.

The specific heat is a purely thermodynamic property and the dependency on the quality of the raw material is almost negligible. In the following analysis, the specific heat of niobium as shown in Fig. 6 is used [11].

The power dissipated on the inner surface of a cavity is transferred to the helium that is in contact with the outer surface of a cavity. When heat is conducted from a solid into liquid helium, a thermal boundary resistance occurs across the interface and consequently there exists a temperature difference $\Delta T$ between helium bath temperature $T_{b}$, and outer surface temperature $T_{s}$. The heat transfer shows dependencies on the phase of helium along with many other factors such as surface conditions.

When using the helium phase-II, it is known to have the following relation [12] for $\Delta T<\sim 1 \mathrm{~K}$ :

$$
q=\frac{C_{K}}{4 T_{b}^{3}}\left(T_{s}^{4}-T_{b}^{4}\right), \quad T_{b}<2.17 \mathrm{~K},
$$

where $q$ is the heat flux across interface in $\mathrm{W} / \mathrm{m}^{2}$, and $C_{K}$ is the Kapitza conductance in $\mathrm{W} / \mathrm{m}^{2} / \mathrm{K}$. The most widely used fit of the Kapitza conductance for SRF cavities made of niobium proposed in [13] is $C_{K}=200 T_{b}^{4.65}$.

The heat transfer to helium phase-I in the nucleate boiling regime, which has more concerns in this paper,

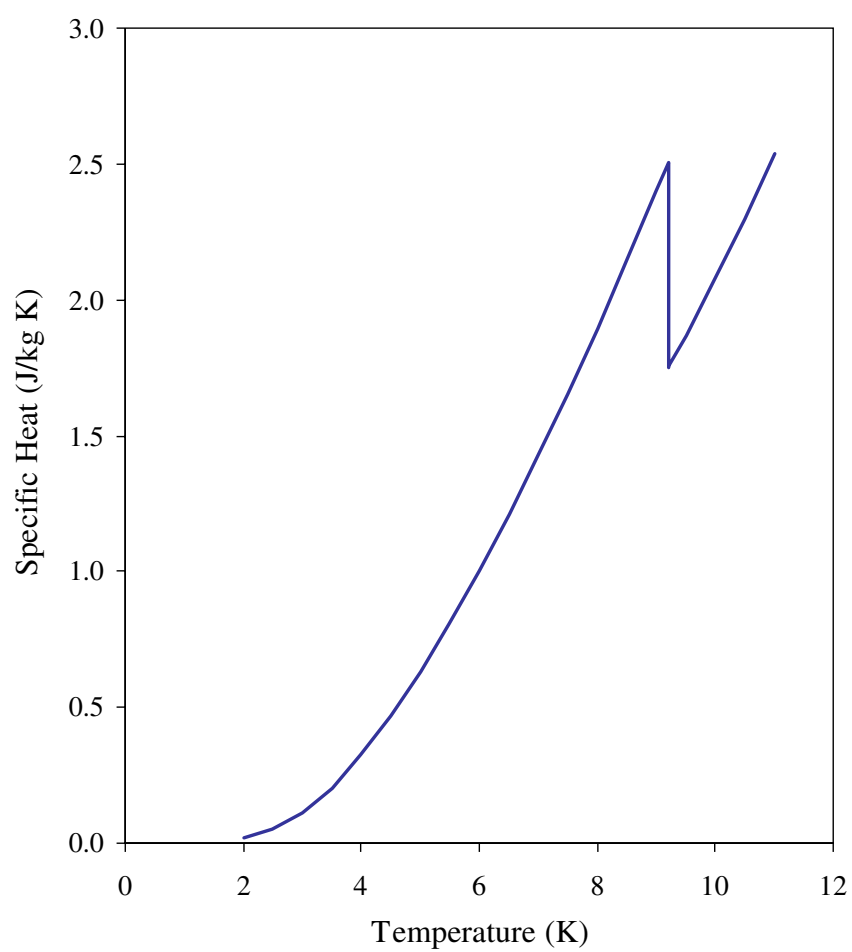

FIG. 6. (Color) Specific heat of niobium at the cryogenic temperature.

has the general form,

$$
q=C\left(T_{s}-T_{b}\right)^{d},
$$

where $C$ and $d$ are constants.

The heat transfer data in this regime show a wide spread $[12,14,15]$. In this study the constants $C$ and $d$ in Eq. (2) are chosen to have reasonable agreement with experimental data [12] to avoid too conservative analysis, since these play deterministic roles for thermal stability. Another stability condition one should take into account is the maximum nucleate boiling flux where film boiling starts, which is known to be about $1 \mathrm{~W} / \mathrm{cm}^{2}$. It is, however, reported that the maximum nucleate boiling flux reduces substantially down to $0.2 \mathrm{~W} / \mathrm{cm}^{2}$ when a surface faces downward [15]. Experimental results also indicate that the nucleate boiling is limited at $0.2 \mathrm{~W} / \mathrm{cm}^{2}$ heat fluxes [16]. In this study, $0.2 \mathrm{~W} / \mathrm{cm}^{2}$ is set as the stability criterion, which would be a primary limiting factor, especially in CW operation. Figure 7 shows heat transfer data for helium phase-I used in this study together with that for helium phase-II shown for comparison.

The computational algorithm in this paper is the same as the structure in Fig. 2, which was developed for the analysis of thermal stabilities of SRF cavities in general purposes [17]. Calculations utilize many merits of wellexplained features of the commercial FEM code (ANSYS) incorporated with particularly developed interfacial programs for steep jumps in material properties, RF surface dissipations in both superconducting and normal states, 


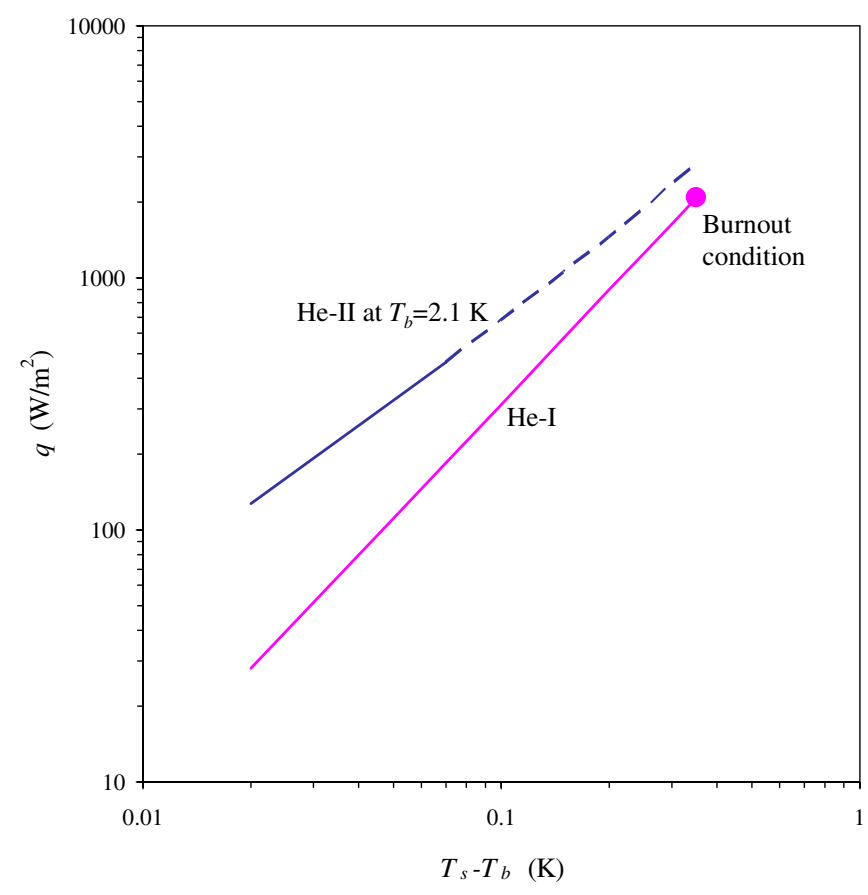

FIG. 7. (Color) Heat transfer density $q$ between helium and the surface of the niobium. $T_{b}$ is the helium bath temperature and $T_{s}$ is the surface temperature of niobium at the helium side. The solid circle at the end of the line for He-I indicates the limit of the nucleate boiling regime.

film boiling condition at the interface between niobium and helium, and RF field conversion to thermal loads. Again, this study is not intended to find absolute numbers in any extreme condition, but to have parameter dependencies of magnetothermal stability on cavity wall thicknesses, helium temperature, RF surface magnetic fields, operating frequencies, pulse lengths, RF duty factors, etc.

\section{B. Comparisons of thermal stability between $\mathrm{CW}$ and pulsed operations}

In CW operation a SRF cavity should reach a stable condition where the heat removal is the same as the RF surface dissipation while keeping, of course, the superconducting state. As can be anticipated from the relations in Fig. 2, especially from the BCS losses (Fig. 4) and the heat transfer at the helium interface (Fig. 7), the intrinsic thermal limitation would come from heat removal to helium in defect-free surfaces. In pulsed operation thermal equilibrium is not necessarily required for stability. This fact implies that a partial quench could be allowable when cavity fields are maintained as required, even though it is not desirable.

Figure 8 shows comparisons between $\mathrm{CW}$ and pulsed operation where an RF surface field of $130 \mathrm{mT}$ at $805 \mathrm{MHz}$ is applied to 4-mm thick niobium having RRR5, as shown in Fig. 5. The helium bath temperature is set to $4.2 \mathrm{~K}$. Both temperature and heat flux reach a steady state at around $20 \mathrm{~ms}$ after RF is applied. The heat flux to helium is about
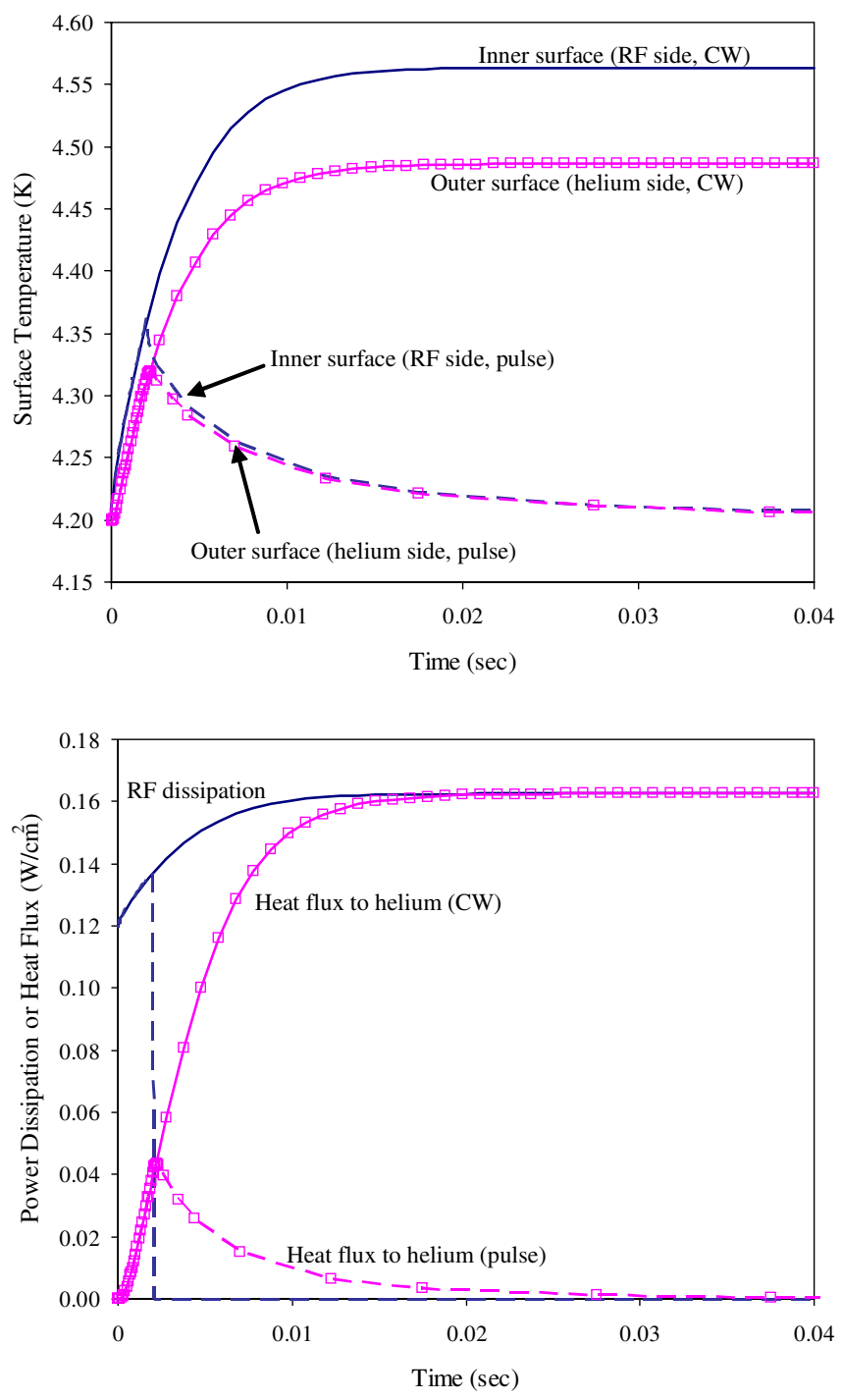

FIG. 8. (Color) Comparisons of thermal parameters between $\mathrm{CW}$ and pulsed operations. Both cases are stable. 130-mT surface magnetic at $805 \mathrm{MHz}$ is applied to 4-mm thick niobium having RRR5 in Fig. 5. The helium bath temperature is set to 4.2 K. 2-ms step RF pulse is used. (solid line, CW operation; dashed line, pulsed operation; lines with symbols, for surface at helium side; lines without symbols, for surface at RF side)

$0.16 \mathrm{~W} / \mathrm{cm}^{2}$ which guarantees the nucleate boiling heat transfer. The differences between inner surface temperature $T_{i}$ and outer surface temperature $T_{s}$ mainly arise from the thermal resistances of niobium; thermal conductivity and wall thickness. In pulsed operation, however, pulse length is usually shorter than the time needed to reach a steady state, so that the temperature increments which determine RF surface dissipation and heat flux to helium during a pulse are less than those in $\mathrm{CW}$ operation. Heat removal to helium is mainly made during a gap. In this example RF pulse length is set to $2 \mathrm{~ms}$.

A thermally unstable case in $\mathrm{CW}$ operation could be stable in pulsed operation as clearly seen in Figs. 9 and 10, 


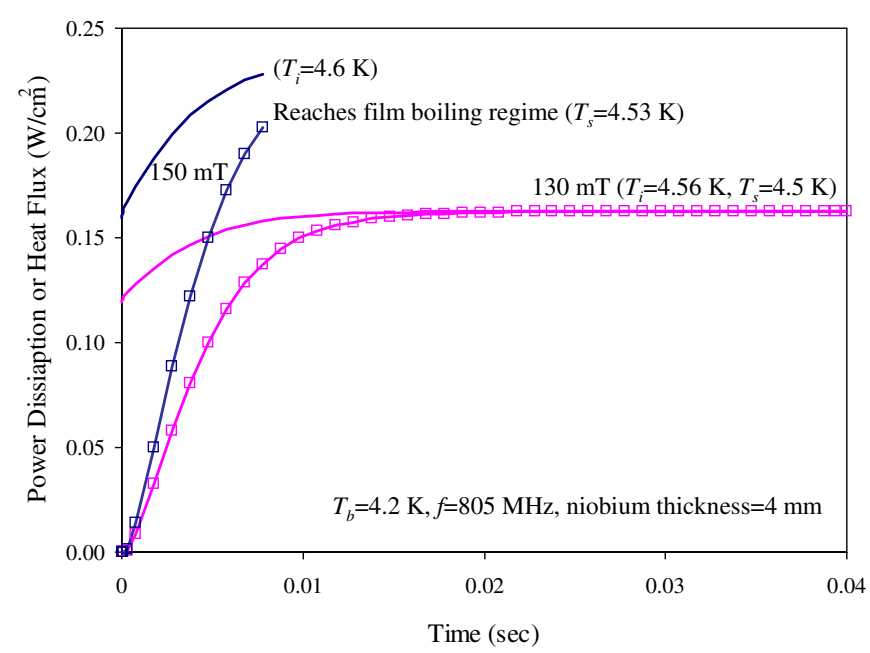

FIG. 9. (Color) RF power dissipations (solid lines) on the RF surface and heat fluxes (lines with symbols) to helium in the CW operation. The heat transfer between the helium and the niobium surface reaches the film boiling regime at $150 \mathrm{mT}$, which is the limiting condition in $\mathrm{CW}$ operation. $T_{b}$ is the helium bath temperature, $T_{i}$ is the niobium surface temperature at the RF side, and $T_{s}$ is the niobium surface temperature at the helium side.

where surface fields at $805 \mathrm{MHz}$ are applied to 4-mm thick niobium having the RRR5 shown in Fig. 5. The helium bath temperature is set to $4.2 \mathrm{~K}$. In Fig. 9 the heat flux at $150 \mathrm{mT}$ reaches the film boiling regime beyond which heat transfer is supposed to be drastically decreased and eventually a cavity quenches. In this example, the RF side

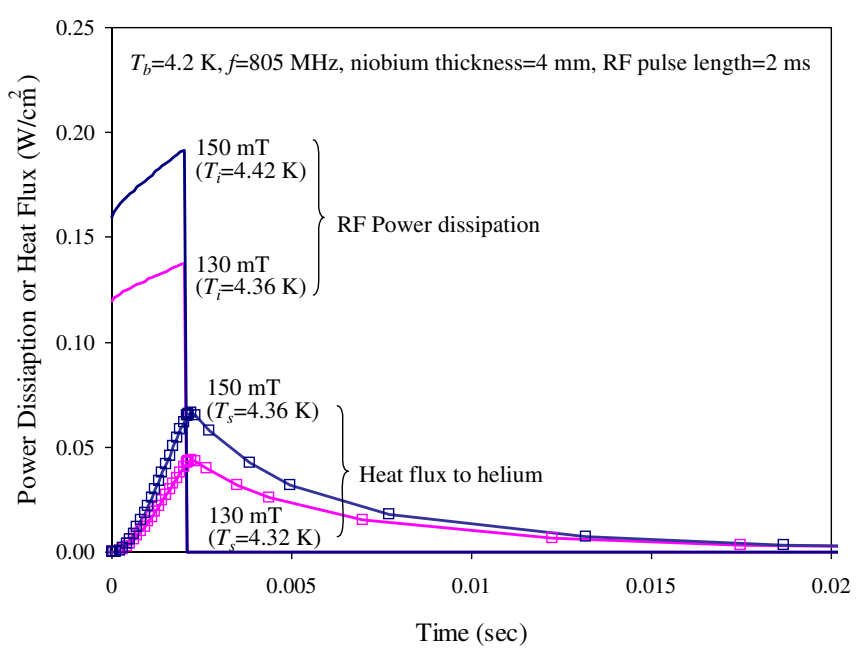

FIG. 10. (Color) Power dissipations (solid lines) on the RF surface and heat fluxes (lines with symbols) to helium in pulsed operation. Operation is stable at the $150-\mathrm{mT}$ surface field where CW operation is limited by the heat transfer to helium (Fig. 9). Temperatures in parentheses are peak values. $T_{b}$ is the helium bath temperature, $T_{i}$ is the niobium surface temperature at the $\mathrm{RF}$ side, and $T_{s}$ is the niobium surface temperature at the helium side. coincidently reaches the temperature where $150 \mathrm{mT}$ is about the critical field. For higher operating frequencies, it is clearly seen that heat flux reaches the film boiling regime much below the critical field. Figure 10 shows the pulsed operation under the same conditions as in Fig. 9 and indicates stable operation up to the critical field at the surface temperature on the RF side.

Another factor to be addressed is the thermal impedance of the solid itself that is characterized by the thermal conductivity and the thickness of niobium. In addition, the specific heat plays an important role in pulsed operation. These effects are shown in the following examples, though variations of thermal parameters either in CW or pulsed operations are interacting with all mechanisms that determine the thermal stability depicted in Fig. 2. Figure 11 plots RF power dissipation and heat flux to helium for various wall thicknesses having the same thermal conductivity (RRR5 in Fig. 5) in CW operation including transient periods. Because of higher thermal impedance, the thicker wall cases reach a thermal equilibrium at a higher inner surface temperature. This results in higher RF power dissipation and also higher thermal flux to helium. In CW operation, a thinner wall is better as long as mechanical concerns are allowable. The very beginning of the transient region in Fig. 11, where heat removal to helium has less contribution, shows a different aspect. In this region, utilization of niobium cold mass dominates, which is usually faster than heat removal to helium up to a certain point. This is, of course, why the slopes of RF power dissipation and accordingly the temperature increment on the inner surface are less for thicker walls. Figures 12 and 13 show the heat flux and temperature increment on both sides of

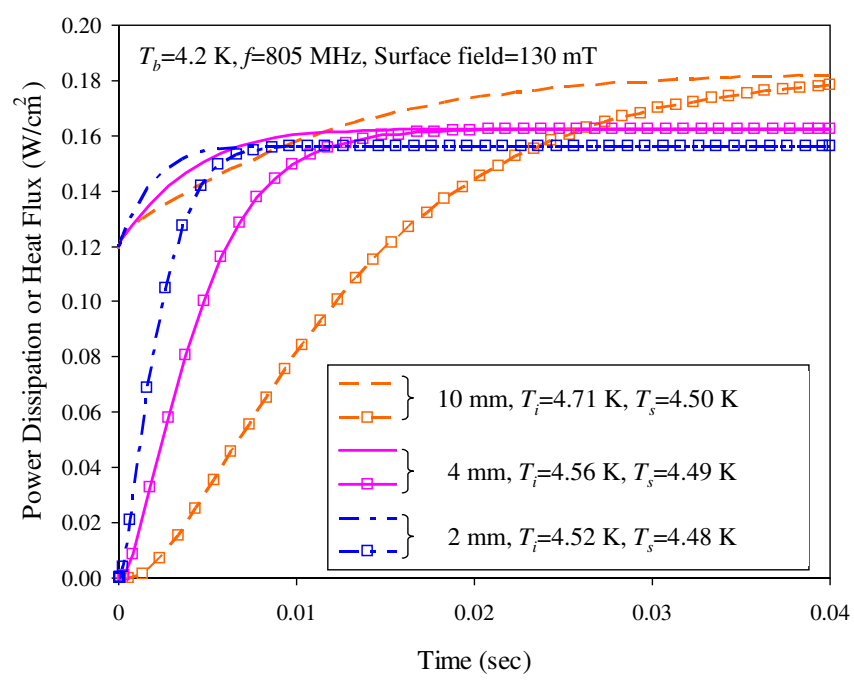

FIG. 11. (Color) Wall thickness dependencies of power dissipations (solid lines) on the surface at the RF side and heat fluxes (lines with symbols) to helium in $\mathrm{CW}$ operation. Temperatures are values in steady state. $T_{b}$ is the helium bath temperature, $T_{i}$ is the niobium surface temperature at the RF side, and $T_{s}$ is the niobium surface temperature at the helium side. 


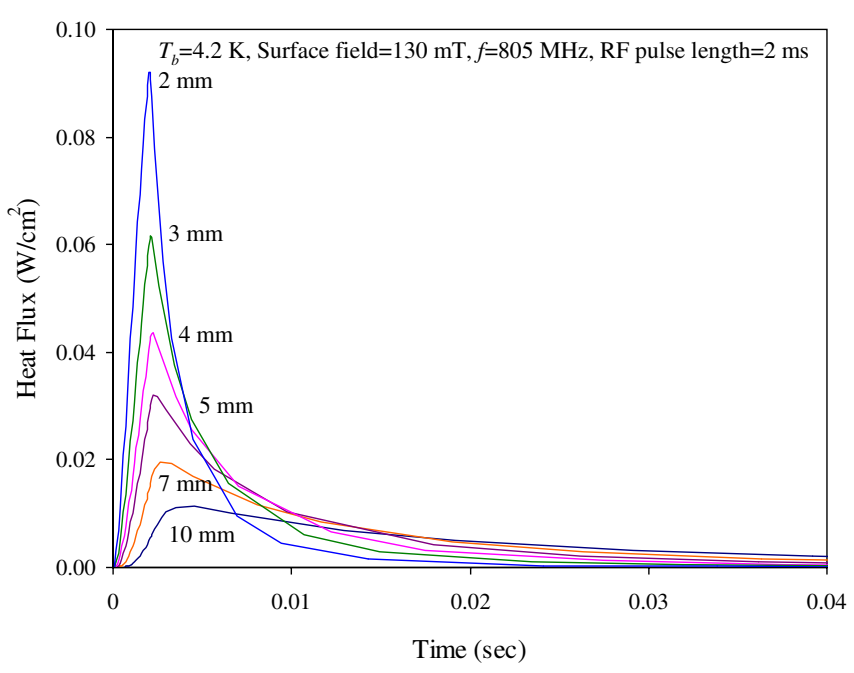

FIG. 12. (Color) Wall thickness dependencies of heat flux to helium in pulsed operation. $T_{b}$ is the helium bath temperature. The thicker shows smaller heat flux to helium and longer recovery time after RF is off.

the wall. Peak temperatures and heat fluxes to helium are higher for thinner walls and thinner walls have a faster recovery during a gap.

When choosing a pulse length and a repetition rate at a given frequency, there is an optimum thickness in terms of thermal stability. For example, a 4-5 mm wall thickness would be optimum in SNS or similar machine that has $2 \mathrm{~ms}$ or shorter pulse length and 5\%-10\% RF duty. Figure 14 shows thermal parameters when $150 \mathrm{mT}$ is applied to 4$\mathrm{mm}$ thick niobium running at $1.5-\mathrm{ms}$ pulse length and

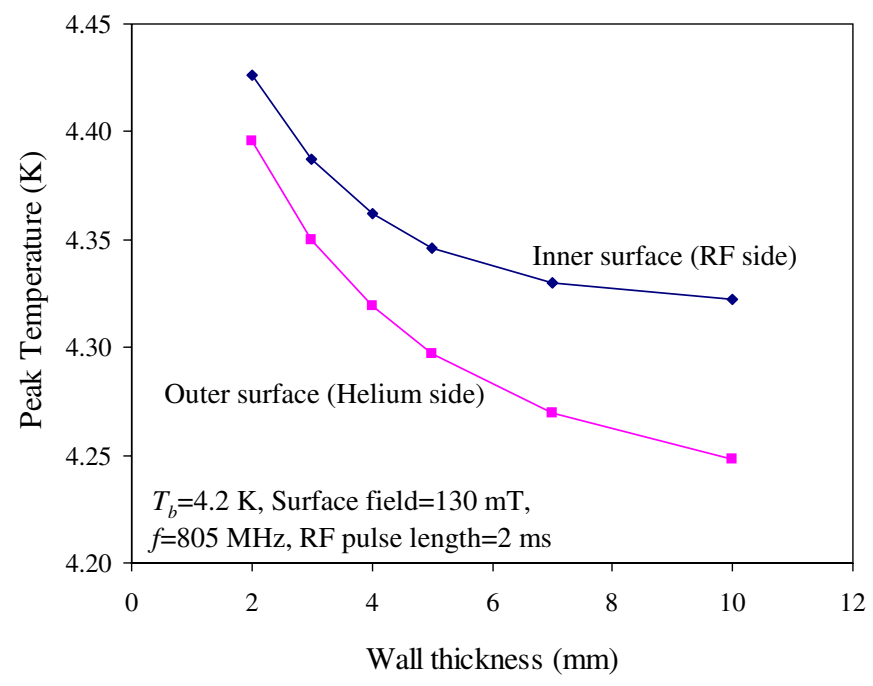

FIG. 13. (Color) Wall thickness dependency of peak temperature in pulsed operation. $T_{b}$ is the helium bath temperature. Temperatures of the niobium surface at the helium side reach their peaks after the end of pulse. Heat transfer to the cold mass is a more efficient way as long as the recovery in the gap is sufficient.
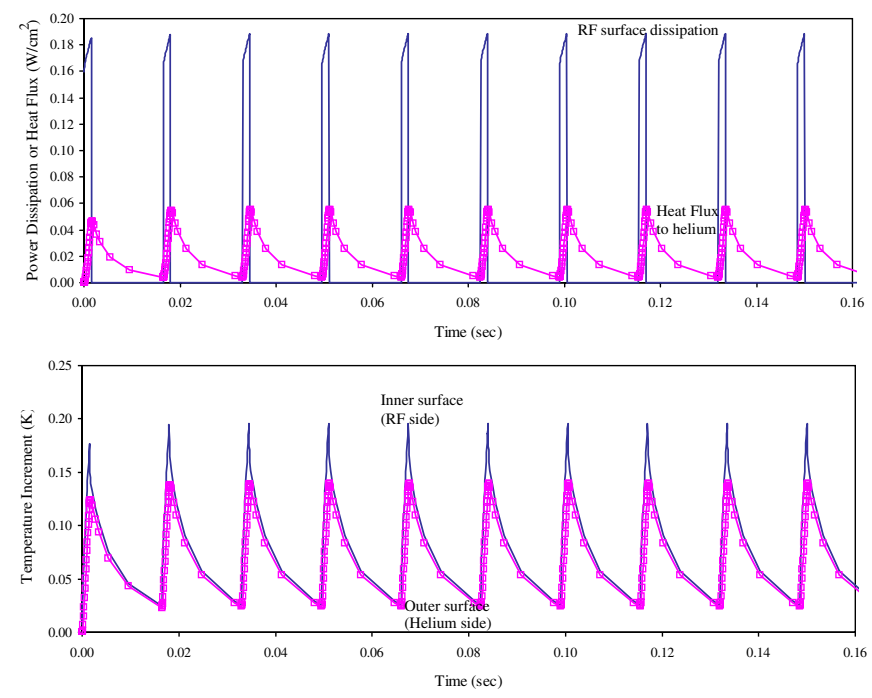

FIG. 14. (Color) RF power dissipation, heat flux (top), and temperature increments on inner and outer surfaces of niobium (bottom) running at $1.5 \mathrm{~ms}, 60 \mathrm{~Hz}$ pulsed operation. $150 \mathrm{mT}$ applied to the inner surface of 4-mm thick niobium and the helium temperature is set to $4.2 \mathrm{~K}$.

$60 \mathrm{~Hz}$, which indicates there is no BCS limitation up to the critical field at this temperature.

As discussed above, CW and pulsed operation have different limiting conditions. In this study, operating parameter space parameters such as frequency, helium bath temperature, RF surface field, niobium wall thickness, pulse length, duty, etc. are scanned to find limiting factors and optimal conditions for both $\mathrm{CW}$ and pulsed operations.

\section{OPTIMIZATION}

\section{A. SNS SCL with the existing cryoplant}

The limiting conditions of $\mathrm{CW}$ operation at $805 \mathrm{MHz}$ are plotted in Figs. 15 and 16. The critical curves of niobium are taken from [18]. The first limiting factor in most cases is the heat flux transition from the nucleate boiling regime to the film boiling regime. Dynamic analyses with the SNS pulse length and duty factor discussed in the previous section show that there are no BCS limitations up to the critical field when the operating temperature is less than $4.5 \mathrm{~K}$. The peak heat flux is less than $0.1 \mathrm{~W} / \mathrm{cm}^{2}$ up to the critical field, which is well below the heat flux limitation even if the same limiting criterion as that for CW operation is applied. That explains the experimental results of the SNS SRF cavities. The best performance of the SNS cavities reached the maximum surface field close to $140 \mathrm{mT}$. This is higher than the CW limitation shown in Fig. 15. When a certain amount of field tilt $(\sim 10 \%)$ is assumed, this value would be very close to the critical field. Consequently, both experimental and analysis results indicate no difference in operation of the SNS SCL between 2.1 and $4.2 \mathrm{~K}$ in terms of thermal stability. 


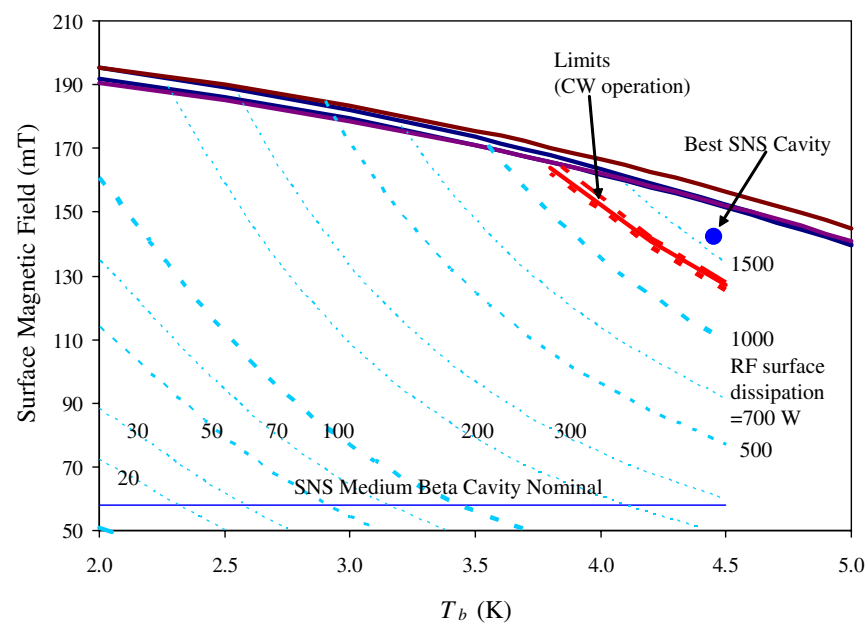

FIG. 15. (Color) Dynamic losses of the SNS medium beta cavity and limits in $\mathrm{CW}$ operation at $805 \mathrm{MHz}$. There are no BCS limitations at the SNS duty and pulse length up to the critical field when $T_{b}<4.5 \mathrm{~K}$. The surface resistance enhancements around the critical field are not taken into account in this analysis, which is beyond of the scope for operating of the SNS SRF cavities. The dashed lines are equisurface dissipations calculated with surface resistances shown in Fig. 3.

Next is the concern of efficiency in operation, which is simplified since limiting fields at the SNS operating condition do not need to be considered at any point up to that of the critical field. The SNS cryoplant was, however, designed for $2.1-\mathrm{K}$ operation, which results in lower efficiency at 4.2-K operation. The conversion factors of the total efficiency from the cryogenic loads to room temperature electricity are about 1200 at $2 \mathrm{~K}$ and 450 at $4.2 \mathrm{~K}$ [19].

Figure 17 is a comparison of operating costs at 2.1 and 4.2 K of the SNS SCL with the existing SNS cryoplant. The

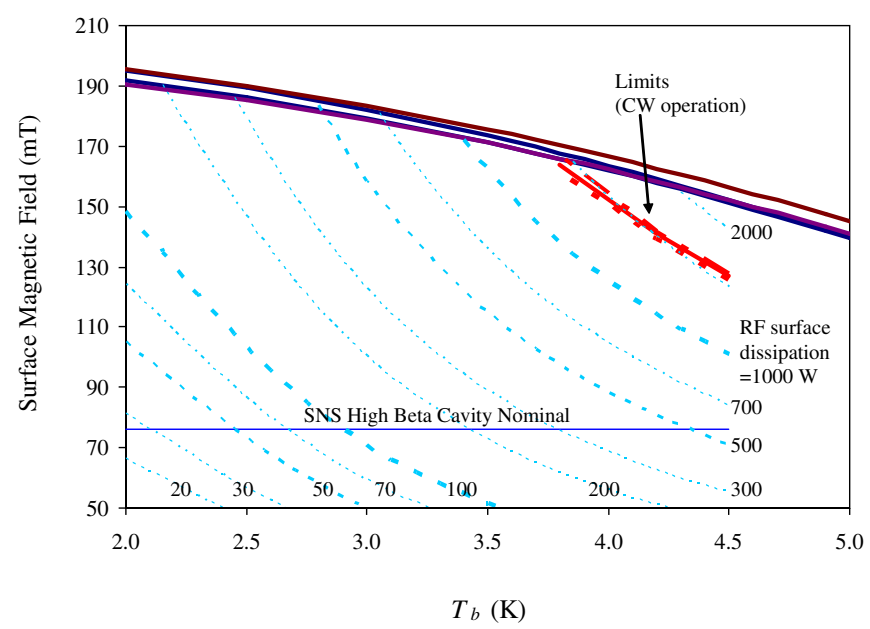

FIG. 16. (Color) Dynamic losses of the SNS high beta cavity and limits in $\mathrm{CW}$ operation at $805 \mathrm{MHz}$. There are no BCS limitations at the SNS duty and pulse length up to the critical field when $T_{b}<4.5 \mathrm{~K}$. Inputs for this plot are the same as those for Fig. 15.

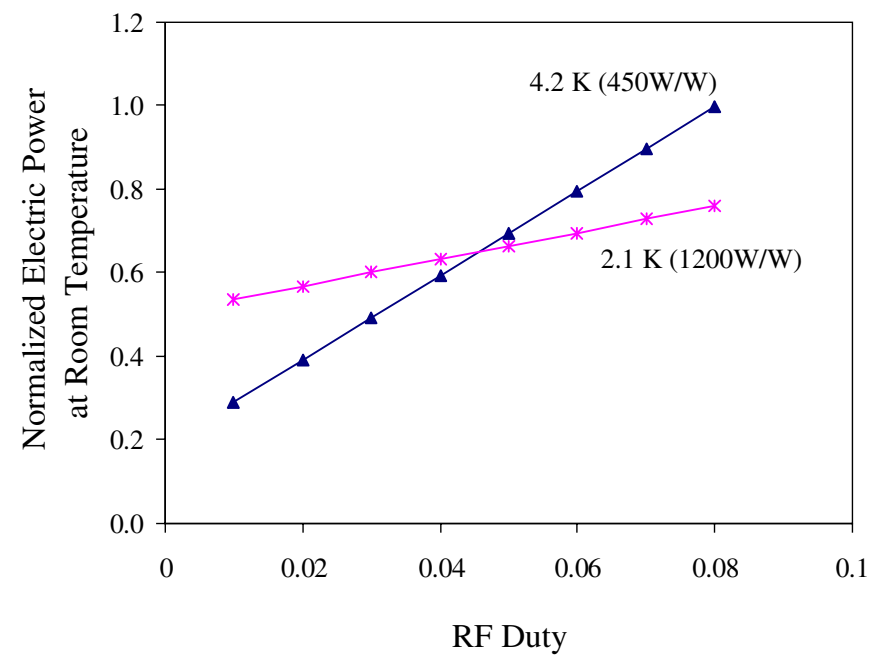

FIG. 17. (Color) Comparison of electric power consumptions of the SNS cryomodules at 2.1 and $4.2 \mathrm{~K}$ with the existing SNS cryoplant at the SNS SCL layout. The total cryogenic efficiencies operating at 4.2 and $2.1 \mathrm{~K}$ are assumed to be 450 and $1200 \mathrm{~W} / \mathrm{W}$, respectively, based on the operational experiences with the existing SNS cryoplant.

static loss of the medium beta cryomodule is about $20 \mathrm{~W}$ and that of the high beta cryomodule is about $30 \mathrm{~W}$ per cryomodule. Also additional dynamic losses are added for field emission, couplers, etc. Even though the SNS cryoplant is designed for $2.1 \mathrm{~K}$, operation at $4.2 \mathrm{~K}$ is efficient when a duty factor is lower as can be seen in Fig. 17. Lower-temperature operation has more merit at a higher duty factor.

\section{B. SNS-like machine with a cryoplant to be designed}

As mentioned in the previous section, there is no BCS limitation at $805 \mathrm{MHz}$ or lower frequencies in pulsed operations having the SNS pulse structure or similar up to the critical field and up to $4.5 \mathrm{~K}$ helium bath temperature. An accelerating gradient and a general layout have a strong impact on the optimization. This factor should, however, be considered in connection with an entire machine where specific machine-dependent parameters play an important role. This factor is not considered in this paper since fair and uniform comparisons of machines are difficult. The following is only a comparison of operating cost on the basis of the SNS SCL parameters and experiences, and assumes a cryoplant to be designed.

Figure 18 shows the total cryogenic loads of the SNS SCL with the same amount of additional dynamic losses as for the plots in Fig. 17. The total cryogenic efficiency is assumed to have the relations in Fig. 19, which is scaled from cryogenic loads and temperatures in Fig. 18. But the total cryogenic efficiency depends on many other parameters and shows a wide spread, so an iterative feedback analysis may be needed in designing actual cryoplants. 


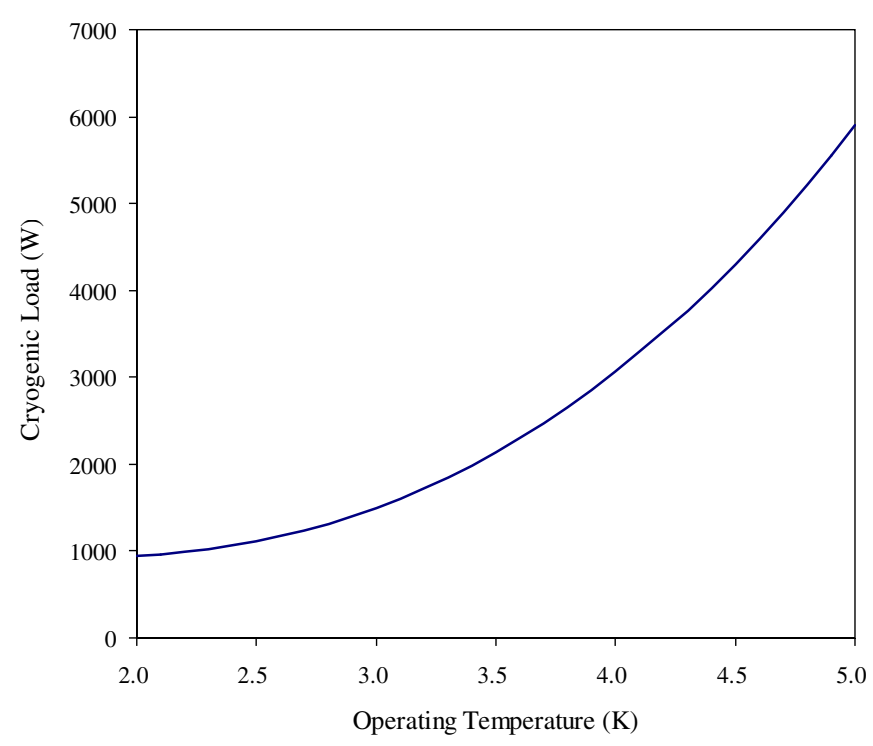

FIG. 18. (Color) Total cryogenic loads on the basis of the nominal SNS SCL parameters.

Relative comparisons of operating costs are estimated from these assumptions and plotted in Fig. 20. As the RF duty factor increases, the optimum operating temperature decreases. The optimum temperature at $8 \%$ duty factor is estimated to be $3 \mathrm{~K}$. The capital cost is not directly included here and cost considerations would likely push the optimum temperature a bit higher. For static losses the SNS

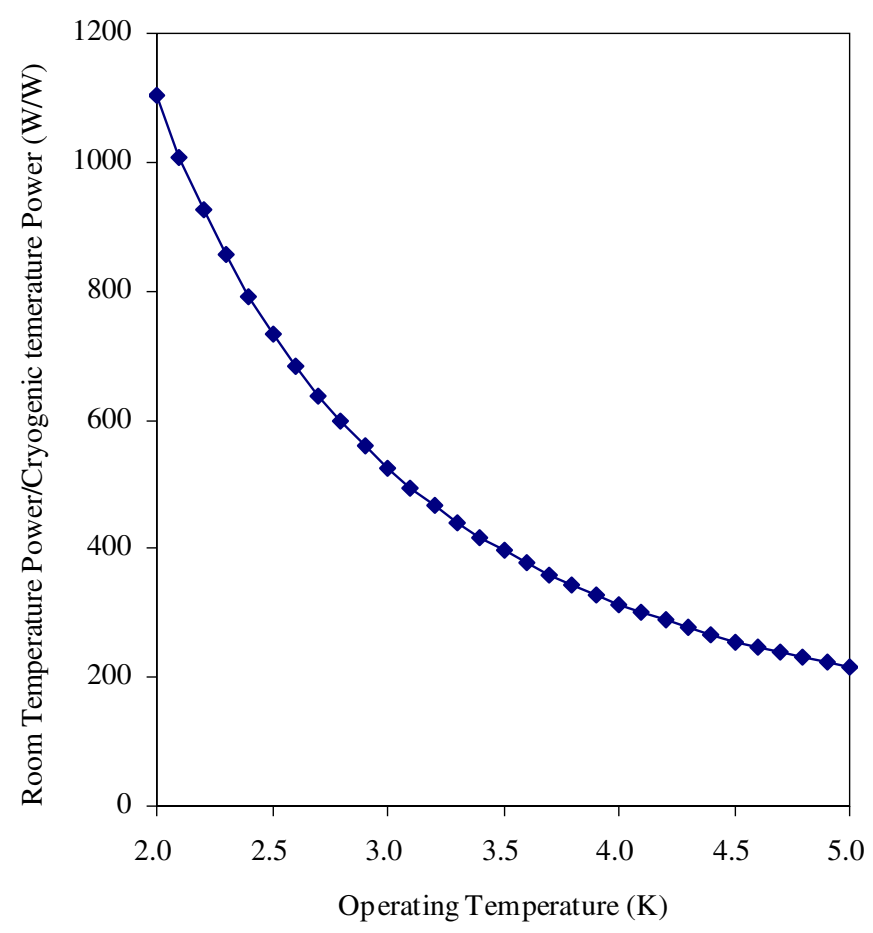

FIG. 19. (Color) Dependencies of total cryogenic efficiency on operating temperature for the calculations of operating efficiencies in Fig. 20.

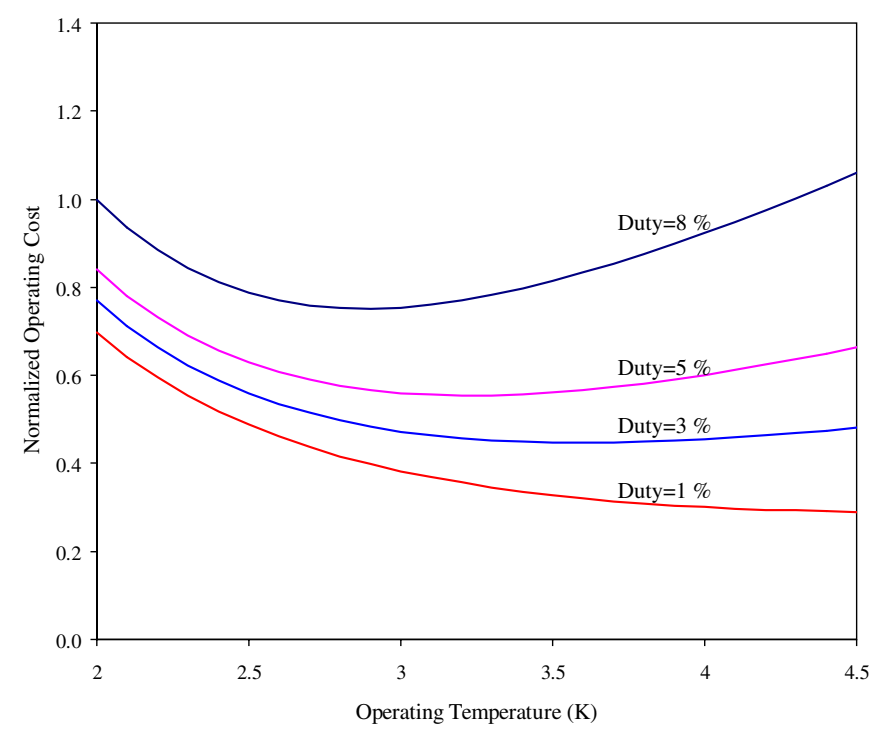

FIG. 20. (Color) Operating cost dependencies on operating temperature and duty factor for an SNS-like machine. Calculations are made with values in Figs. 18 and 19.

values are assumed, but cryostats with lower static losses than SNS will again decrease the optimum temperature.

\section{DISCUSSIONS ON OTHER FREQUENCIES}

Analyses are expanded to some other frequencies since the frequency dependency is one of the major factors in determining thermal stability, as shown in Fig. 4. Limitations in CW operation are compared in Fig. 21. Each frequency has three lines that correspond to wall thicknesses; the top one to 3-mm thick, the middle one to 4-mm thick, and the bottom one to 5-mm thick. As explained previously, the thicker walls have less thermal stability in CW operation. In most cases, cavities are first tested in the vertical test assembly in CW operation. Figure 21 indicates that the achievable field drastically decreases as the operating temperature increases, especially at higher frequencies. For example, about $20 \mathrm{MV} / \mathrm{m}$ is the maximum achievable accelerating gradient in a $1300 \mathrm{MHz}$, TESLA-type cavity when it is operated at $4.2 \mathrm{~K}$ in $\mathrm{CW}$. Figure 21 also shows that the $700 \mathrm{MHz}$ or less frequency region has no BCS limitation up to a critical field at $4.2 \mathrm{~K}$, even in $\mathrm{CW}$ operation.

Gains of thermal stability in pulsed operations at higher frequencies are more clearly seen than in $805 \mathrm{MHz}$ cases shown in previous sections. Figure 22 plots the limitations of operation for $1300 \mathrm{MHz}$ and Fig. 23 those for $1000 \mathrm{MHz}$. In pulsed operation a partial quench would be allowable as long as the cavity accelerating field is not affected and cavities could be kept in a superconducting state for a certain amount of time after heat flux exceeds this criterion. The same criterion for CW stability is, however, applied to pulsed operation; when the heat flux to helium reaches $0.2 \mathrm{~W} / \mathrm{cm}^{2}$, the system is assumed to be 


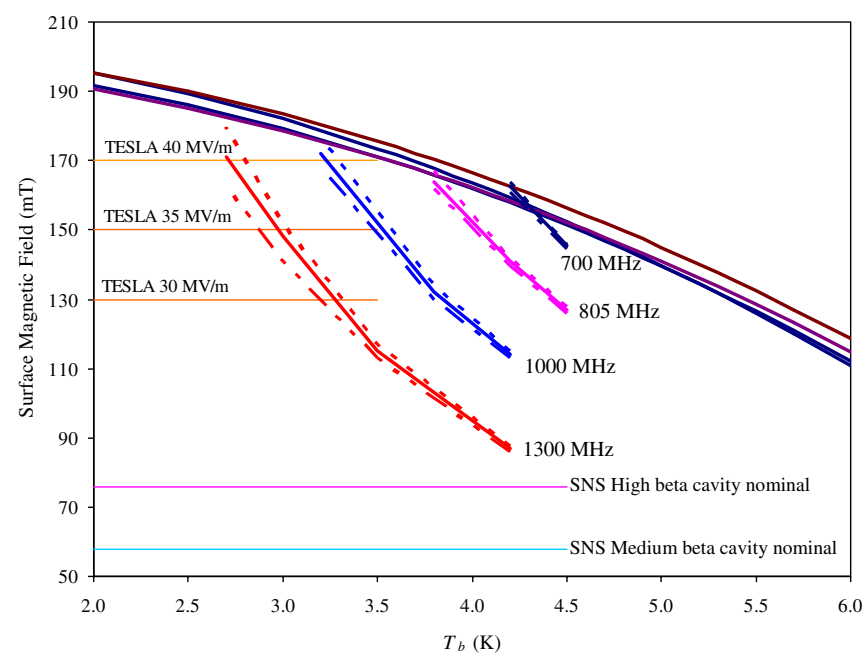

FIG. 21. (Color) Limitations in CW operation for various operating frequencies. The limiting factor is mainly heat transfer to helium when it reaches the film boiling regime. For comparison surface fields of the SNS nominal ones $(805 \mathrm{MHz})$ and the TESLA baseline cavity $(1300 \mathrm{MHz})$ at different accelerating gradients are plotted together. $T_{b}$ is the helium bath temperature. Thinner niobium wall thickness has more stability margin in CW operation. Three lines for each frequency correspond 2-mm, 3$\mathrm{mm}$, and 4-mm niobium wall thickness from the top.

unstable. The thickness of niobium plays an important role. It does not mean that the thicker one is always better, since the optimum thickness for thermal stability is related to pulse length and duty factor. For the frequencies, pulse lengths, and duty factors discussed in this paper, the best advantage would be given with a thickness of $4-5 \mathrm{~mm}$.

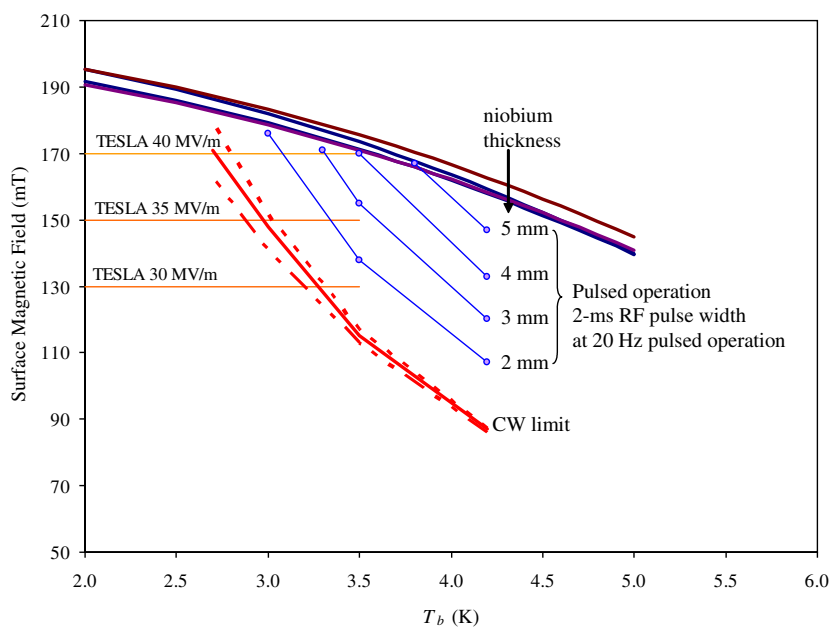

FIG. 22. (Color) Limitations at $1300 \mathrm{MHz}$ in $\mathrm{CW}$ and pulsed operations. $T_{b}$ is the helium bath temperature. The thicker wall up to $5 \mathrm{~mm}$ has more thermal stability in this example of pulsed operation at $2 \mathrm{~ms}, 20 \mathrm{~Hz}$, while the thinner one has more thermal stability margin in $\mathrm{CW}$ operation. Three lines for the $\mathrm{CW}$ limit correspond 2-mm, 3-mm, and 4-mm niobium wall thickness from the top.

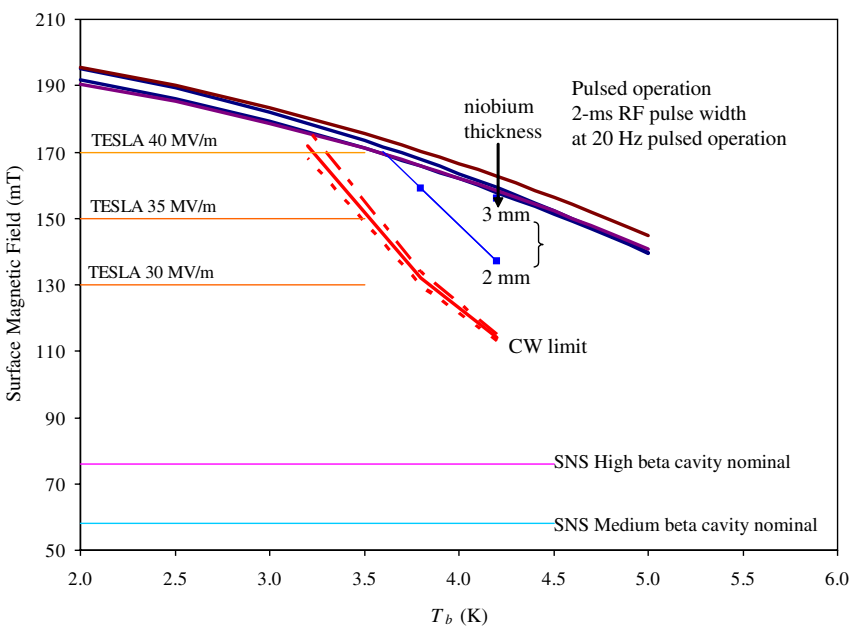

FIG. 23. (Color) Limitations at $1000 \mathrm{MHz}$ in $\mathrm{CW}$ and pulsed operations. $T_{b}$ is the helium bath temperature. There are no BCS limitations in this example of pulsed operation at $2 \mathrm{~ms}, 20 \mathrm{~Hz}$ when the niobium is thicker than $3 \mathrm{~mm}$ and helium bath temperature is less than $4.5 \mathrm{~K}$. Three lines for the $\mathrm{CW}$ limit correspond 2-mm, 3-mm, and 4-mm niobium wall thickness from the top.

The next step would be a cost optimization for pulsed operations. It is difficult to find general sets of optimized cost parameters, since many factors are machine specific. For example, the ratio between static and dynamic losses is very important for optimization when a duty factor is small. The static loss is determined by cryostat design, cryogenic layout, etc., and the dynamic loss depends on duty factor, frequency, and the operating field that is related to machine length, construction cost, cryogenic loads at a specific operating temperature, etc. Relative comparisons are possible. A higher-temperature operation is relatively beneficial with higher contributions from static loss, lower accelerating gradient, lower duty factor, and lower frequency. It would be interesting to postulate a frequency range of about $1000 \mathrm{MHz}$ for the $\beta=1$ low loss structure with a very low duty factor, since there is no BCS limitation up to the critical field in pulsed operation and a low loss structure could be designed to have $35 \mathrm{MV} / \mathrm{m}$ accelerating gradient with a margin even at $4.2 \mathrm{~K}$.

\section{CONCLUSIONS}

The thermal stabilities of pulsed operation are explored in the parameter space of operating temperature, surface field, frequency, niobium thickness, pulse length, and duty factor and are compared with those of $\mathrm{CW}$ operation. The transient nature of pulsed operation allows larger variation of temperatures within the niobium surface, so that during the pulse the surface resistance of the material can change substantially.

The operating conditions of the SNS SRF cavities were reviewed and cavity parameters and their interplay were reevaluated, giving details on the possible range of oper- 
ating parameters which can be achieved at SNS in pulsed conditions. Though the nominal operating gradients of the SNS SRF cavities are relatively low, the cavities can be operated at $4.2 \mathrm{~K}$ up to the critical field, due mainly to the pulsed nature of the SNS operation and the relatively low operating frequency.

Suggestions for optimization are given for the SNS SCL operation and for the design of future SNS-like machines. At low duty factor operation of the SNS SCL, 4.2-K operation results in a lower operating cost, even with the SNS cryoplant that is not optimized for operation at $4.2 \mathrm{~K}$.

Similar studies for other frequencies are made. Limiting conditions are estimated in both $\mathrm{CW}$ and pulsed operations, which we hope would assist in evaluating optimal operating parameters of superconducting cavities at other frequencies.

\section{ACKNOWLEDGMENTS}

The authors extend our thanks to all our ASD/SNS colleagues who contributed to this work, especially to Fabio Casagrande for the fruitful discussions on cryogenic systems. SNS is managed by UT-Battelle, LLC, under Contract No. DE-AC05-00OR22725 for the U.S. Department of Energy. SNS is a partnership of six national laboratories: Argonne, Brookhaven, Jefferson, Lawrence Berkeley, Los Alamos, and Oak Ridge.

[1] N. Holtkamp, in Proceedings of Twenty Second International Linear Accelerator Conference, 2004, p. 837.

[2] C. Rode, in Proceedings of the 2001 Particle Accelerator Conference, 2001, p. 619.

[3] D. S. Stout, I. E. Campisi, F. Casagrande, R. Cutler, D. Hatfield, M. Howell, T. Hunter, R. Kersevan, P. Ladd, and H. Strong, in Proceedings of the 2005 Particle Accelerator
Conference, 2005, p. 3838.

[4] I. Campisi and Z. D. Farkas, Stanford Linear Accelerator Center, SLAC-PUB-3617, 1985.

[5] I. Campisi, in Ref. [3], p. 34.

[6] I. Campisi, in Proceedings of Twelfth Workshop on RF Superconductivity, 2005, MoA02.

[7] G. Ciovati, P. Kneisel, G. Myneni, and W. Lanford, in Proceedings of Eleventh Workshop on RF Superconductivity, 2003, WeO14.

[8] H. Padamsee, J. Knobloch, and T. Hays, $R F$ Superconductivity for Accelerators (John Wiley \& Sons, New York, 1998).

[9] D. Reschke, in Proceedings of Eighth Workshop on RF Superconductivity, 1997, p. 385.

[10] M. Fouaidy, in Proceedings of Ninth Workshop on RF Superconductivity, 1999, TUP028.

[11] R. P. Reed and A. F. Clark, Materials at Low Temperature (American Society for Metals, Ohio, 1983).

[12] R. ballantini, A. Chincarini, G. Gemme, and R. Parodi, Phys. Rev. ST Accel. Beams 6, 083201 (2003).

[13] K. Mittag, Cryogenics 13, 94 (1973).

[14] M. Wilson, Superconducting Magnets (Clarendon Press, Oxford, 1983).

[15] R. Smith, in Proceedings of the 1968 Summer Study on Superconducting Devices and Accelerators, edited by A. Prodell (Brookhaven National Laboratory, New York, 1968).

[16] E. Kako, S. Noguchi, M. Ono, K. Saito, T. Shishido, H. Inoue, T. Fujino, Y. Funahashi, M. Matsuoka, T. Higuchi, T. Suzuki, and H. Umezawa, in Proceedings of Tenth European Particle Accelerator Conference, 1996, p. 2124.

[17] Sang-ho Kim, in Proceedings of the 2003 Particle Accelerator Conference, 2003, p. 1365.

[18] K. Saito, in Ref. [7], MoO02.

[19] F. Casagrande, P. Gurd, D. Hatfield, M. Howell, and H. Strong, in Proceedings of Tenth European Particle Accelerator Conference, 2006, p. 514. 